\title{
Light-Controlled, Toehold-Mediated Logic Circuit for Assembly of DNA Tiles
}

Chao Xing†t, Ziyi Chen†, Junduan Dai†, Jie Zhou†, Liping Wang†, Kai-Long Zhang†, Xiaofei Yin§, Chunhua Lu*† and Huanghao Yang*†

$\dagger$ MOE Key Laboratory for Analytical Science of Food Safety and Biology, Fujian Provincial Key Laboratory of Analysis and Detection Technology for Food Safety, State Key Laboratory of Photocatalysis on Energy and Environment, College of Chemistry, Fuzhou University, Fuzhou 350116, P. R. China.

$\$$ Fujian Provincial Key Laboratory of Functional Marine Sensing Materials, Center for Advanced Marine Materials and Smart Sensors, Minjiang University, Fuzhou 350108, P. R. China.

$\S$ First Institute of Oceanography, Ministry of Natural Resources, Qingdao 266061, P. R. China.

*E-mail: chunhualu@fzu.edu.cn (C. L.).

*E-mail: hhyang@fzu.edu.cn (H. Y.) 


\section{Results and Discussion}

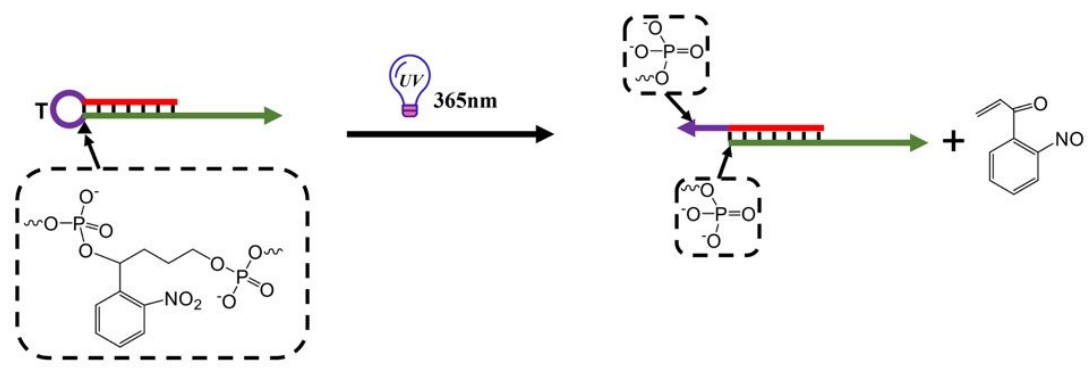

Figure S1. Scheme of light-controlled toehold T formation system.

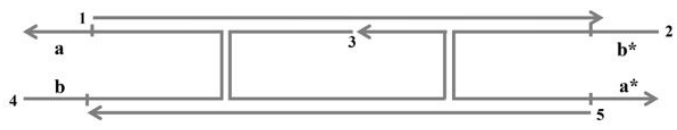

Tile

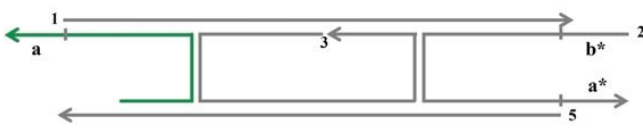

IT 1

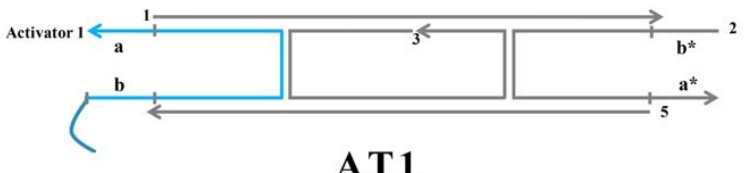

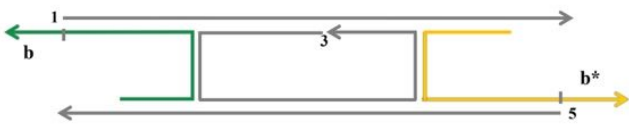

I T 2

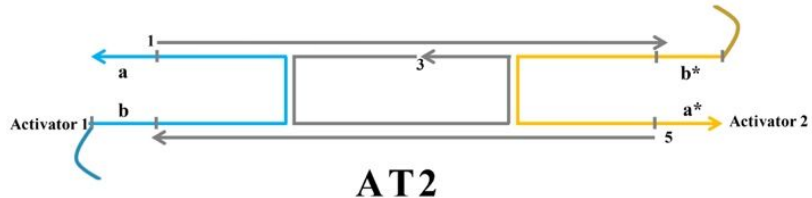

AT2

Figure S2. Complete, incomplete and activated tile. In incomplete tile, the missed sticky end (IT1, a; IT2, a and a*) lead to stable, monomeric tiles that cannot self-assemble.

$\mathbf{a}$

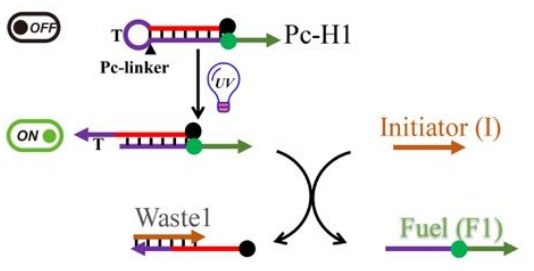

b

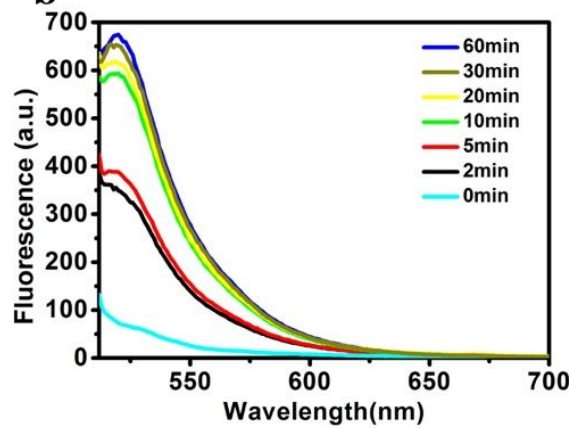

c

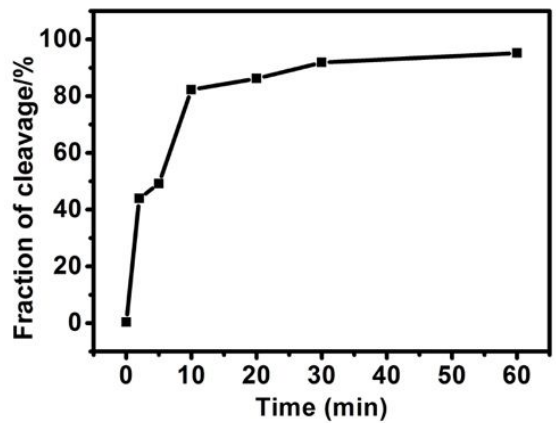

Figure S3. (a) Fluorescence characterization of light-controlled hidden-toehold activation. (b) Fluorescence test of light-controlled DNA strand displacement reaction with different irradiation times. Fluorescence experiment was performed by adding Initiator (I), $200 \mathrm{nM}$ to the solution of Pc-linker modified DNA hairpin $(200 \mathrm{nM})$ with different UV irradiation time, and react $3 \mathrm{~h}$ at room temperature. (c) The fraction of cleavage of the hairpin in different irradiation time. 


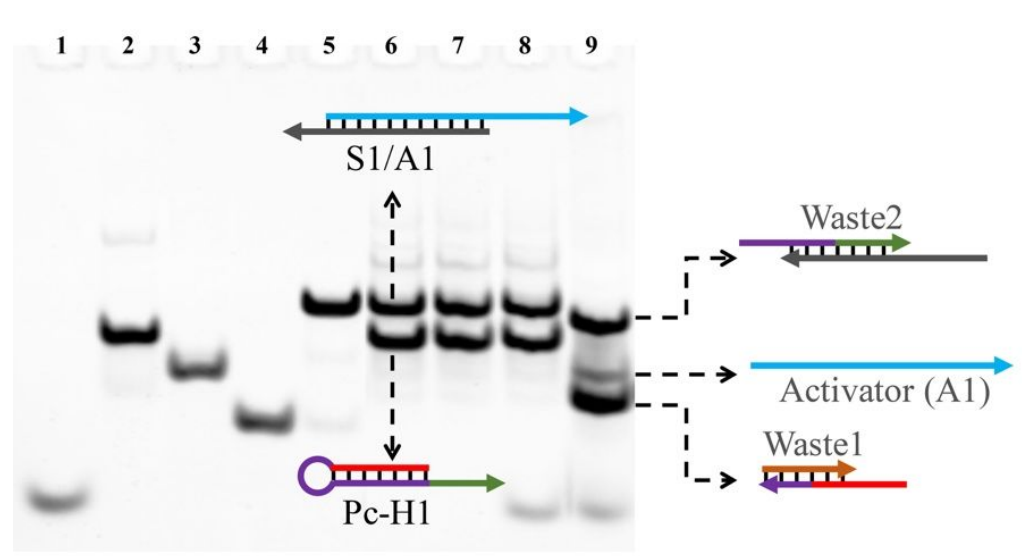

Figure S4. Native PAGE (10\%) analysis of the light-controlled, toehold-mediated DNA circuit. Lane 1: I; lane 2: Pc-H1; lane 3: A1; lane 4: S1; lane 5: duplex of S1/A1; lane 6: Pc-H1 (without irradiation) + duplex of S1/A1; lane 7: Pc-H1 (with irradiation) + duplex of S1/A1; lane 8: I + Pc-H1 (without irradiation) + duplex of S1/A1; lane 9: I + Pc-H1 (with irradiation) + duplex of S1/A1.

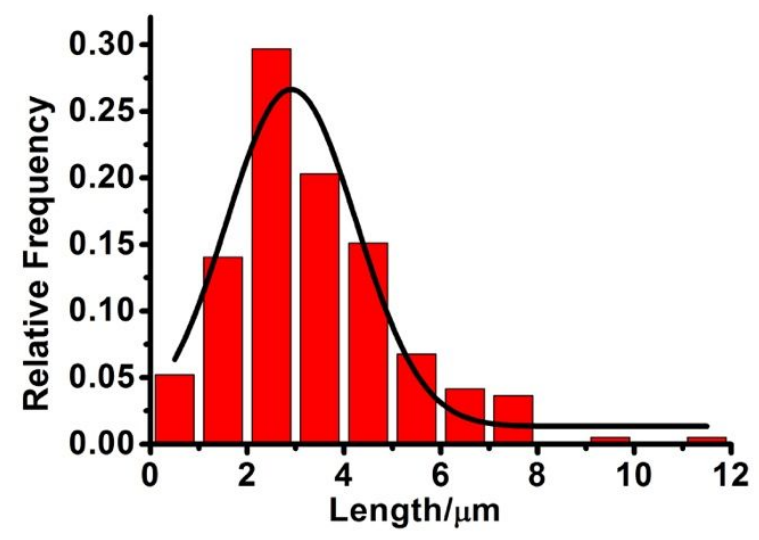

Figure S5. Statistical analysis of assembled tiles length by light-controlled circuit.

\section{$\mathbf{a}$}

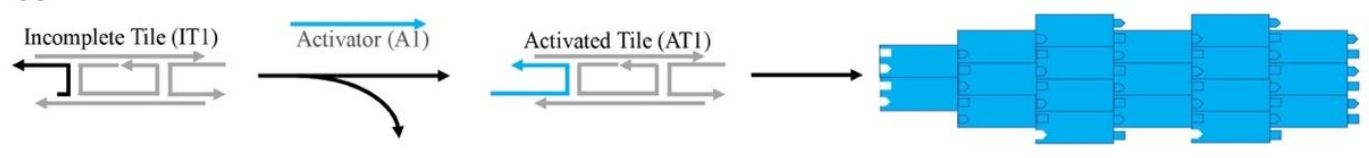

b

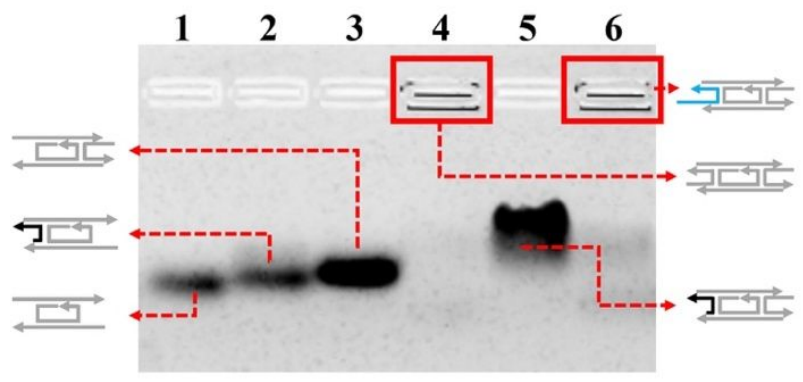

C
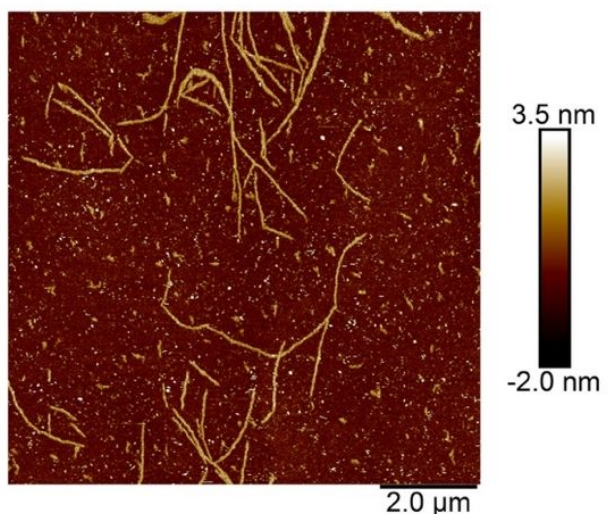

Figure S6. Light-independent DNA tile self-assembly reaction. (a) As a control system, the activator strand (A1) was exogenously added to the solution of incomplete Tiles (IT1). (b) Analysis of DNA tile self-assembly by agarose gel. Lane 1: DNA tile with T1, T3, T5 strands; lane 2: DNA tile with T1, T3, T5, T2i strands; lane 3: DNA tile with T1, T3, T4, T5 strands; lane 4: complete tile self-assembly; lane 5: IT1; lane 6: IT1 + A1; (c) AFM imagine of the above control experiment after the adding of activator strand. 
a
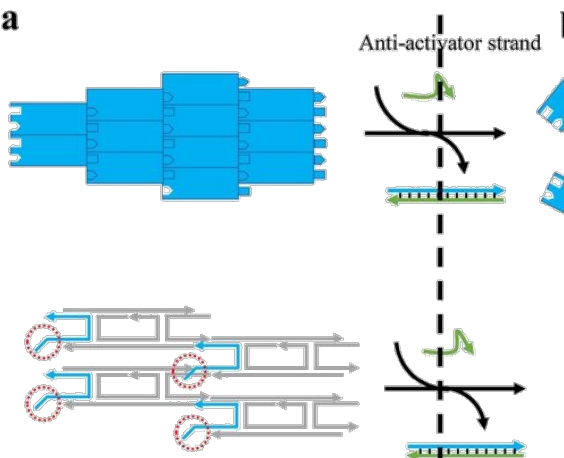

b

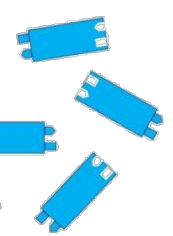

Activator strand
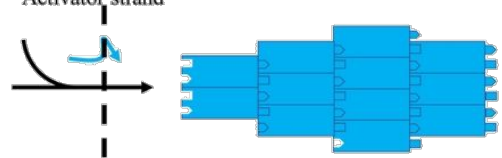

(1)

I
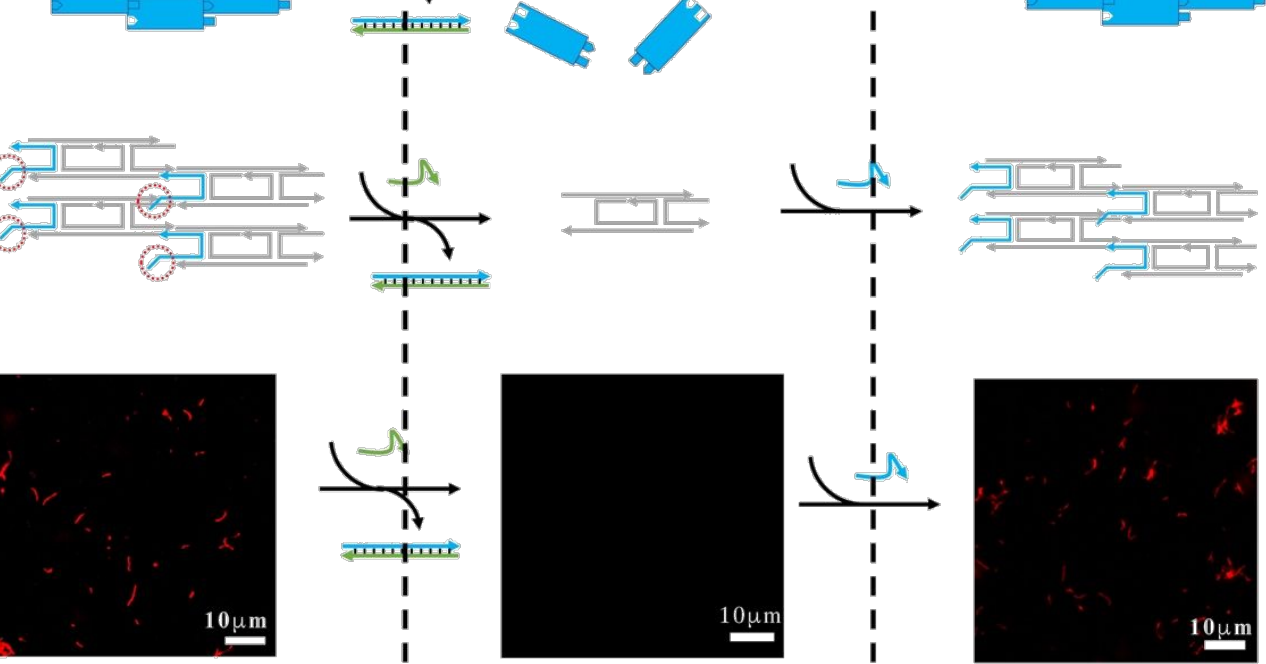

Figure S7. (a) The assembly of the DNA nanotube. (b) Disassembly of the DNA nanotube by anti-activator strand. (c) The re-assembly of the DNA nanotube by re-adding activator strand.
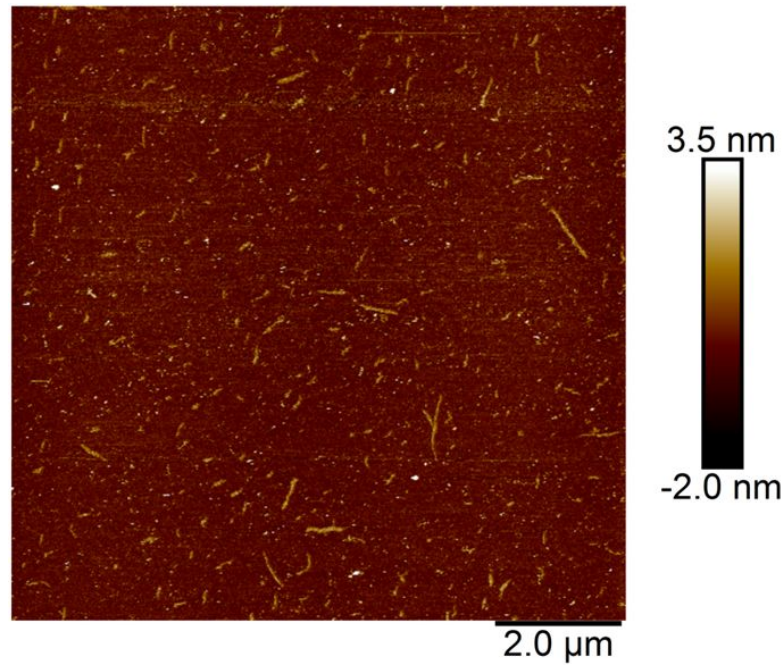

Figure S8. AFM characterization after 2 days of incubation without UV irradiation. The assembly of small lattices is observed under AFM after 2 days without UV irradiation, we think it presumably due to the leak of the activator 1 from the substrate.

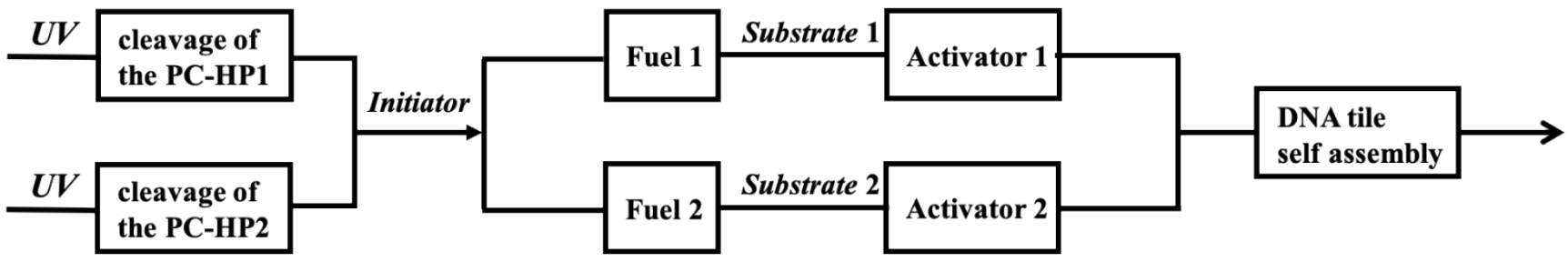

Figure S9. Schematic illustration of the AND gate. 

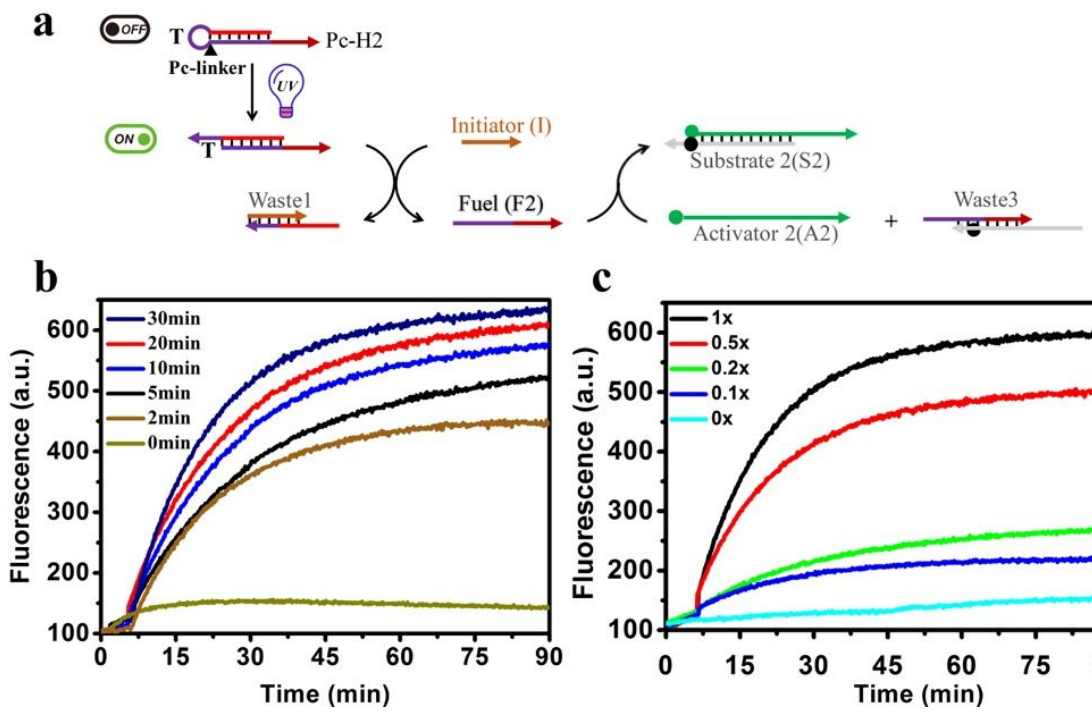

c

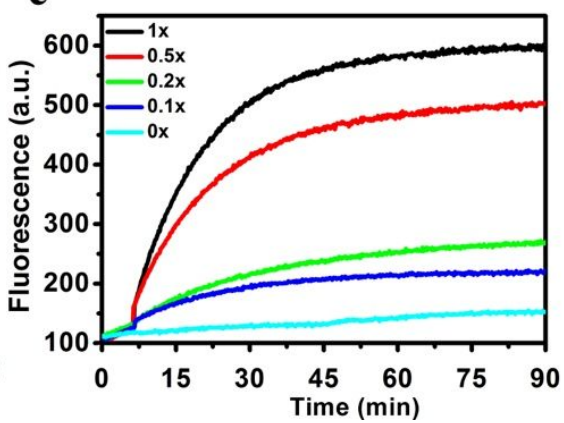

Figure S10. (a) Upstream light-dependent Pc-H2 DNA fluorescence circuit. (b) Fluorescence test of light-controlled Pc-H2 path with different irradiation times. (c) Fluorescence assay of the kinetics of Activator 2 (A2) release at different initiator ratios. The experiments were performed using the following concentrations of reagents: Pc-H2, $200 \mathrm{nM}$; initiator (I), $200 \mathrm{nM}$; FAM and Dabcyl-labeled substrate (S2), $200 \mathrm{nM}$.

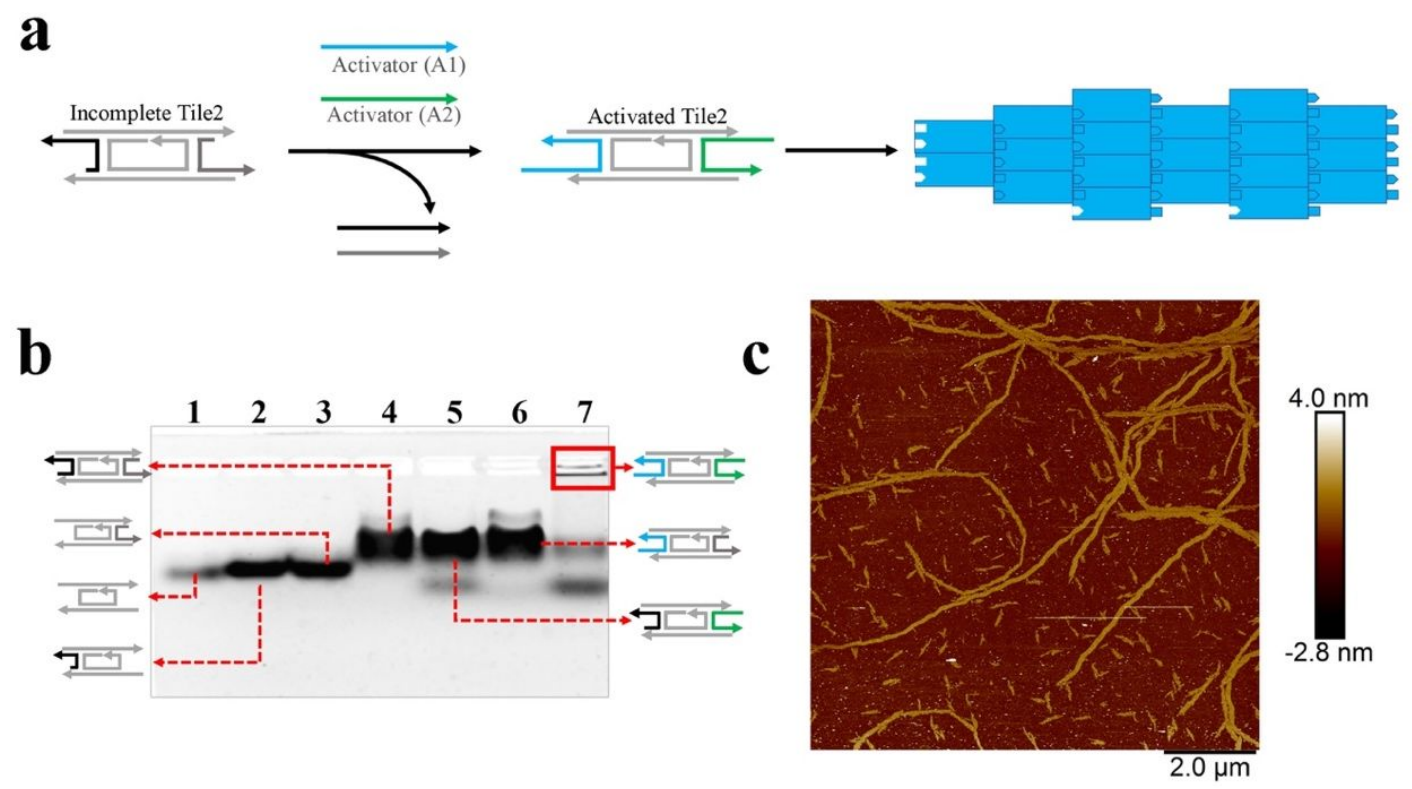

Figure S11. (a) The demonstration of the feasibility of the downstream AT2 self-assembly process. (b) Analysis of the feasibility of the downstream AT2 self-assembly process by agarose gel. Lane 1: DNA tile with T1, T3, T5 strands; lane 2: DNA tile with T1, T3, T5, T2i strands; lane 3: DNA tile with T1, T3, T4i, T5 strands; lane 4: IT2; lane 5: IT2 + A1; lane 6: IT2 + A2; lane 7: IT2 + A1 + A2. Only upon addition of both the activator 1 and activator 2, monomer tiles (lane 7) migrated with higher molecular weight (red box). (c) AFM imagine of the above control experiment after the adding of activator strand. 


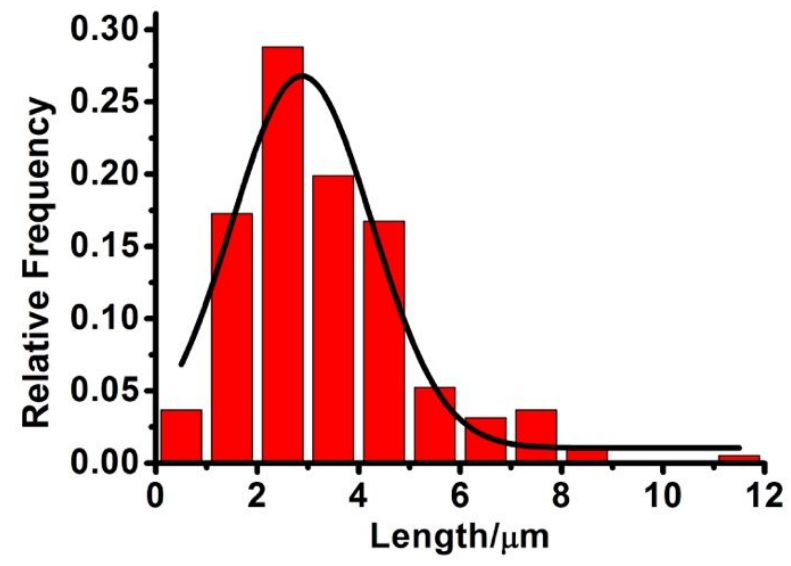

Figure S12. Statistical analysis of assembled tiles length by AND gate.

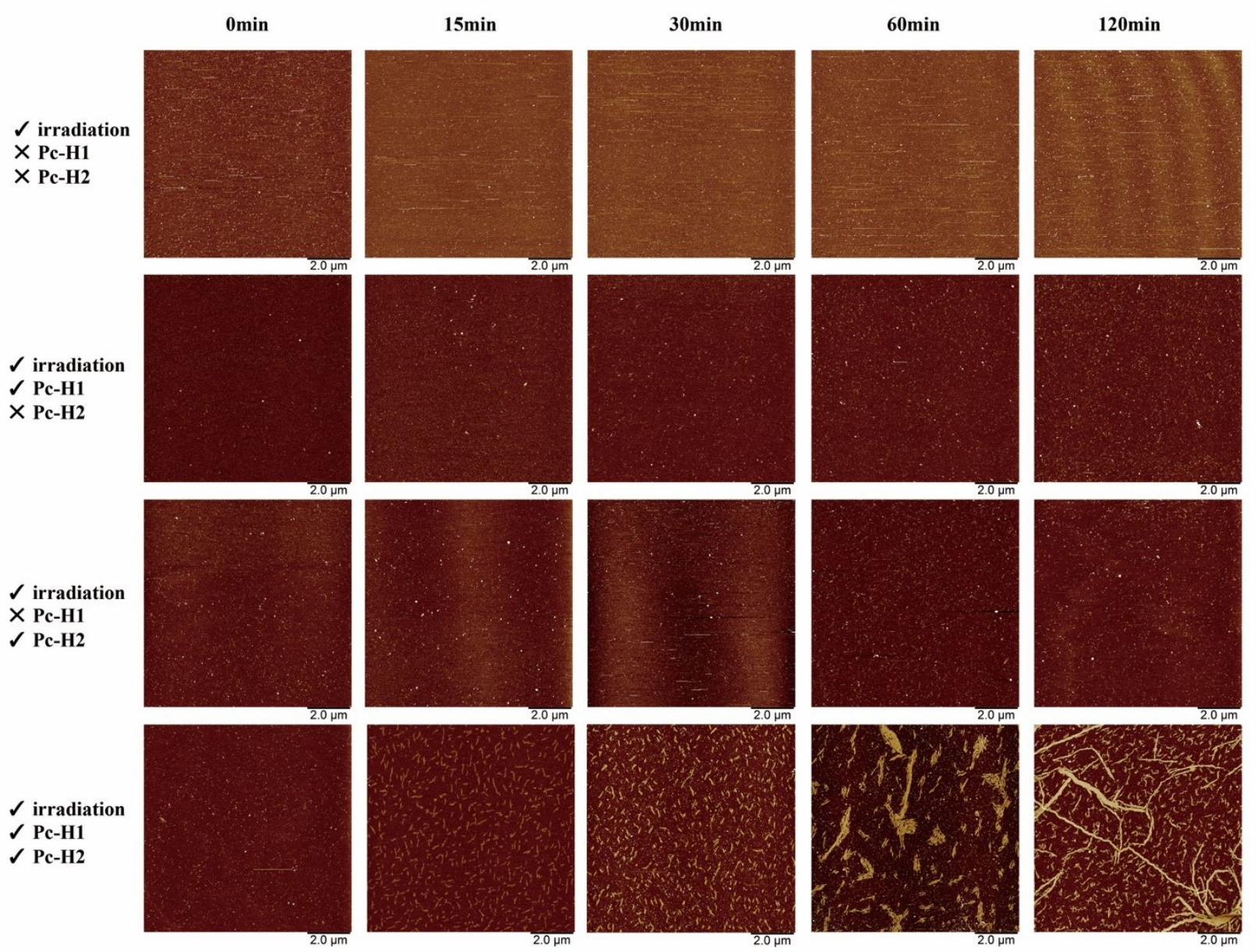

Figure S13. Kinetics of AND gate controlled self-assembly of DNA tiles. Time course AFM images of nanotubes during the incubation of the sample at $25{ }^{\circ} \mathrm{C}$. No tile assembly was observed during the imagining time of the experiment at the condition of no initiator or no irradiation. In contrast, with the present of initiator and irradiation, DNA nanotubes can be clearly observed at $30 \mathrm{~min}$. 
Table S1. The DNA strands used in this study.

\begin{tabular}{|c|c|}
\hline Initiator 1 & GCAGCTCGTTCTCTA \\
\hline $\begin{array}{l}\text { PC-linker DNA } \\
\text { hairpin H1 }\end{array}$ & $\begin{array}{l}\text { TCGACTAGAGAACGAGCTGC/PC-Linker/ } \\
\text { TCGTTCTCTAGTCGATTAATGCGCAGAC }\end{array}$ \\
\hline Activator 1 & TTAATGCGCAGACAGTTTCGTGGTCATCGTACCT \\
\hline Anti-activator1 & AGGTACGATGACCACGAAACTGTCTGCGCATTAA \\
\hline Substrate 1 & AAACTGTCTGCGCATTAATCGAC \\
\hline FAM-Activator 1 & 6-FAM-TTAATGCGCAGACAGTTTCGTGGTCATCGTACCT \\
\hline Dabcyl-Substrate 1 & AAACTGTCTGCGCATTAA/Dabcyl/TCGAC \\
\hline Fluorescence-H1 & $\begin{array}{l}\text { Dabcyl-TCGACTAGAGAACGAGCTGC/PC-Linker/ } \\
\text { TCGTTCTCTAGTCGA/FAM/TTAATGCGCAGAC }\end{array}$ \\
\hline FAM-Fuel & TCGTTCTCTAGTCGA/FAM/TTAATGCGCAGAC \\
\hline $\mathrm{T} 1$ & CTCAGTGGACAGCCGTTCTGGAGCGTTGGACGAAACT \\
\hline $\mathrm{T} 2$ & GTCTGGTAGAGCACCACTGAGAGGTA \\
\hline T3 & CCAGAACGGCTGTGGCTAAACAGTAACCGAAGCACCAACGCT \\
\hline $\mathrm{T} 4$ & CAGACAGTTTCGTGGTCATCGTACCT \\
\hline T5 & CGATGACCTGCTTCGGTTACTGTTTAGCCTGCTCTAC \\
\hline $\begin{array}{l}\text { T2-imcomplete } \\
\text { (T2i) }\end{array}$ & GCACCACTGAGAGGTA \\
\hline $\begin{array}{l}\text { T4-imcomplete } \\
\text { (T4i) }\end{array}$ & CGTGGTCATCGTACCT \\
\hline $\begin{array}{l}\text { PC-linker DNA } \\
\text { hairpin } \mathrm{H} 2\end{array}$ & $\begin{array}{l}\text { TCGACTAGAGAACGAGCTGC/PC-Linker/ } \\
\text { TCGTTCTCTAGTCGATTAATGCGGTCTG }\end{array}$ \\
\hline Activator 2 & TTAATGCGGTCTGGTAGAGCACCACTGAGAGGTA \\
\hline Substrate 2 & TCTACCAGACCGCATTAATCGAC \\
\hline FAM-Activator 2 & 6-FAM-TTAATGCGGTCTGGTAGAGCACCACTGAGAGGTA \\
\hline Dabcyl-Substrate 2 & TCTACCAGACCGCATTAA/Dabcyl/TCGAC \\
\hline
\end{tabular}

